\title{
A Research and Application of Three Dimensional Digitization Technology for Restore Chinese Architectural heritage from the Thirteen Factories
}

\author{
Qionglin Liu',a, Jun Chen ${ }^{2}$ and Yuhua Chen ${ }^{3}$ \\ ${ }^{1}$ Head of Environment Design Department, School of Art and Design, Guangzhou University of Finance and Economics, 510320, China \\ ${ }^{2}$ Arch PhD, The Third Branch Institute Dean of Guangzhou Zoneteam Architectural Design \& Urban Planning Institute Co.,Ltd, China \\ ${ }^{3}$ Electronic \& information major graduate student, École, Nationale Supérieure de l'électronique et de ses applications (ENSEA), France
}

Keywords: Digitization technology; Non-physical historical architecture; the Thirteen Factories; Virtual reproduction

\begin{abstract}
The purpose of this paper is to study Non-physical historical buildings composed by computer 3D models to enhance the experience of city culture and realize the "digital city" information management. Non-physical historical buildings could be an important core cover a city culture and memory, and main competitiveness of city development as well. The Thirteen Factories of canton was the center of Sino-Western commercial which including the integration of culture and trade. The research methodology mainly adapted on three approach. 1) Interpretation and transformation for historical information at the Thirteen Factories Yi Guan Area. 2) According to 3D modeling and texture processing achieve real virtual recreation practice.3) To discuss the ways of digital technology to restore the Non-physical historical buildings. This paper focuses on relationship of digital technology and historical culture heritage, which could improve the economics transformation of city culture.
\end{abstract}

\section{Research background}

Nowadays, computer graphics, digital image processing and virtual reality and other digital restoration and reproduction technologies are developing rapidly and widely which are used in education, military, aviation, medicine, robotics, commerce and so on. They have not only changed the way of production around the world, but also brought enormous social economic benefits to the world. Digital technology has become a synonym for the information age, representing the creation of intelligence and the development of new knowledge, unlimited information acquisition and information exchange. Information could be produced and stored in a digital and electronic way. It is closely integrated with various production factors such as knowledge, capital, technology, and management. It spreads and flows around the world through the network, forming a new productivity under the new normal.

Digitization provides advanced means and methods for the inheritance and protection of architectural cultural heritage. The development of technologies such as Three Dimensional (3D) modeling technology, 3D scanning technology, and computer storage technology has promoted the research and application of digitization of historical architecture information. As a way to build a virtual world by using virtual reality system and reproduce the lost cultural heritage of human in real time, given its basic characteristics of immersion, interaction and imagination. ${ }^{2}$ Compared with the cultural heritage of physical architecture, non-physical historical architecture has no real material existence or remains. Through the interpretation and data transformation of the records and documents of non-physical historical architecture, the lost historical architectural cultural heritage can be made into visual virtual products for people to learn, communicate and innovate.

\section{Analyse of history information}

"The Thirteen Factories (in Mandarin, Shisan Hang or the Canton Factories) in Qing Dynasty" refers to the Chinese foreign trade system established in Guangzhou in Qing Dynasty, which is different from Ming Dynasty "Tribute Trade System". This monopoly system of Chinese and foreign trade has gone through the three dynasties of Qianlong, Jiaqing and Daoguang, concentrating the rise and fall of the Qing Empire and creating Thirteen Factories cultures of architecture, painting, export and poetry. It has led the unprecedented integration and development of Chinese and Western marine commercial culture, formed the wisdom crystallization of the development and

\footnotetext{
${ }^{\text {a }}$ Corresponding author: L@gdufe.edu.cn.

2 Seen in Burdea G. \& Philippe Coiffet.Virtual Reality System and Application[C]. 1993 World Electronics Annual Conference, 1993.
} 
progress of the entire human civilization, and reached a new height of cultural exchanges between the East and the West. The Thirteen Factories Yi Guan Area ${ }^{3}$ originally located on the north bank of the Pearl River and the west of Canton City (Figure 1), is the architecture physical form of the Thirteen Factories of Canton with an area of about 30,000 square meters (Figure 2). The Yi Guan Area is Economic and Cultural growth and pole of the Eighteenth and Nineteenth centuries in the Pearl River Estuary. There are no building left, only some Spatial morphology of them can still be seen outside the old Guangzhou city. However, the Thirteen Factories Culture and pan-culture have the significance of sustainable heritage tourism development. The spatial form heritage is also an important part of Guangzhou's urban trade history and cultural development.

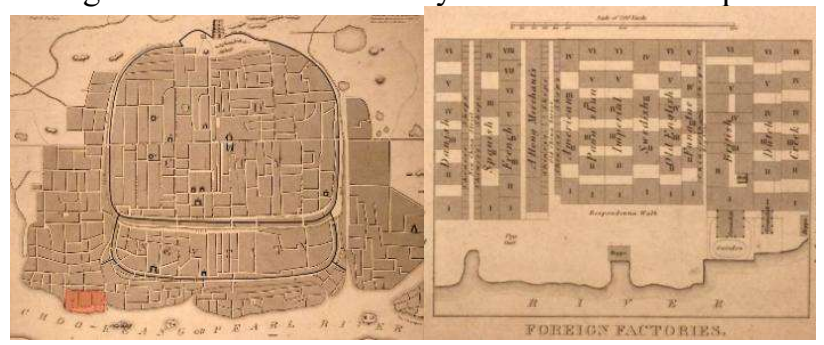

Figure 1 (Left) The Location of the Thirteen Factories of Canton in $1840^{4}$

Figure 2(Right) The distribution plan of the Thirteen Factories in Map of Canton $1840^{5}$

Combining with the practice of 3D digitization of $\mathrm{Yi}$ Guan Area in Qing Dynasty, this paper discusses the digitization technology and application of non-physical historical architecture. The digitization results of this study can be directly applied to the digitization preservation, tourist attraction navigation, virtual interactive system of historical architecture of the Yi Guan Area in Qing Dynasty, etc., as well as provide theoretical support and case reference for informational data acquisition methods for the digitization of similar historical architecture.

\section{The key of 3D digitization restore}

The 3D digitization process of non-physical historical architecture consists of three steps: 3D information acquisition and interpretation, 3D modeling and texture processing. 3D modeling includes scene modeling and image modeling. Compared with the physical historical architecture, the non-physical historical architecture can only be seen in historical documents, painting historical materials, poetry and legend. In the entire digitization process of historical architecture, we will encounter the following problems: This historical architecture only have two-dimensional image information and text descriptions.

\footnotetext{
${ }^{3}$ The Thirteen Factories Yi Guan Area: The Business and Trade Area of the Thirteen Factories of Canton

${ }^{4}$ Map of Canton 1840. Hong Kong Museum of Art. View of the Pearl River Delta: Macau, Guangzhou and Hong Kong[M]. Printing Department, 2002.P143.

${ }^{5}$ Ibid.
}

There are few questions should be focus, the image classification of different time, the transformation of digital data, the topographic and geomorphologic information in different time periods and restore the texture of building. These have gathered knowledge of humanities, social sciences, natural sciences and engineering technology. Therefore, the 3D digitization of non-physical historical architecture has the following three characteristics: (1) Authenticity: For example, in Qing Dynasty, Yi Guan Area of Canton exist from 1685 to 1856, photography was invented in 1839, and only a few Thirteen Factories of black-and-white photographs remain. ${ }^{6}$ Moreover, it is impossible to collect data of nonphysical historical architecture by modern digital photography or 3D laser scanning. The authenticity of 3D information and data can only rely on the accurate interpretation of images and words. From the cultural heritage site and scene to interpret, it needs to be grasped from the overall height. (2)Accuracy: The texture of the modeling needs to express the exquisite decoration of historical architecture and the texture of redundant materials. In the absence of a live image, the accuracy of the material texture needs to be verified. At the same time, the architectural heritage in the $3 \mathrm{D}$ virtual interactive system needs to express the true spatial size relationship. ${ }^{7}$ (3) Repeatability: Historical architecture and their environments include various types of components such as dome arches, pediment, stigmas, porch, railings, figures, boats, etc., and many models need to be constructed. Because of the variety of components, a standard model base can be established and used repeatedly.

In view of the above three characteristics, based on the existing research and acquired image and text information, we gradually derive the time period and spatial nodes of the digitization of Yi Guan Area, and examine the relevant images and documents one by one. On this basis, we have drawn up the digital flow chart of Yi Guan Area in Canton in Qing Dynasty (Figure 3).

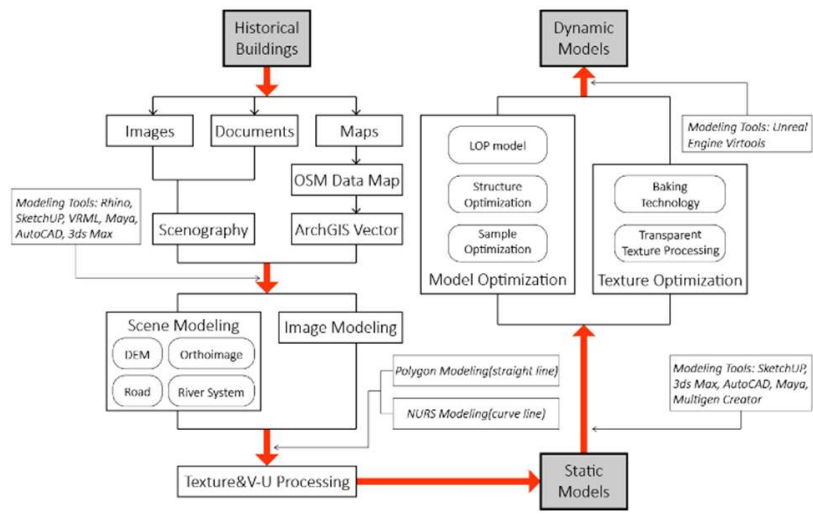

Figure 3. The digital flow chart of the Yi Guan Area

\footnotetext{
${ }^{6}$ In 1844, the Frenchman Yule Eger (1802-1877) photographed a British Pavilion on the Thirteen Factories Doulan Street.He is currently the first foreign photographer recognized by the academic community to engage in photography activities in China.

7 In the history of China, the maps of the measured topographic maps were drawn into various scales. The map of Huangfu was completed in the "The Emperor's Full Picture" in the 47th to the 57th year of Qing Emperor Kangxi (1708 1718).
} 


\subsection{D Information Acquisition}

The digitization of historical architecture is to establish a virtual interactive system and connect it with a digital city information system. Therefore, virtual reality consists of two parts: One part of it is the virtual world created (environment), and the other part is the intervener ( with person). The virtual world is a spatial feature within a certain range above and below the surface of the geographical area, a natural topographic surface such as a lake vegetation, a human landscape such as a building road, and underground pipelines and geological structures. The two parts contain environmental information and architectural information. 3D virtual architecture modeling is one of the key technologies of virtual reality technology. The quality of the modeling directly affects the quality of the $3 \mathrm{D}$ virtual interactive system. The mature computer software used to build the virtual architecture model includes AutoCAD, 3ds Max, SketchUp, Maya, Rhino, VRML and so on. Information on non-physical historical architecture could be collected through literature collection, site surveys, questionnaire surveys, and expert visits on architecture history, age identification, overall planning, form features, construction techniques, architecture materials, historical events, and architecture value assessments. Through amount of data analysis and integration, the conclusion is reached. The following is a case study of environmental modeling, which explains how to interpret, identify and acquire document information.

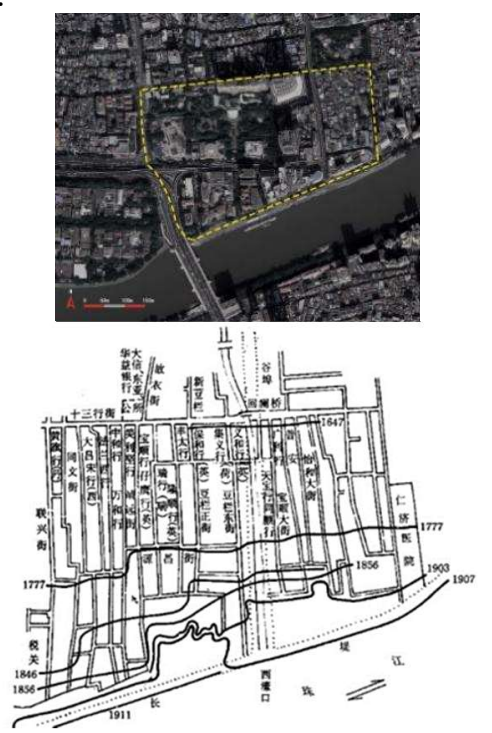

Figure 4 (Left) Yi Guan Area geographic graph in 2018

\footnotetext{
${ }^{8}$ Zeng zhaoxuan（曾昭璇），Guangzhou Geography History [M]. Guangzhou: Guangdong People pubulish house, 1991.

${ }_{9}$ In 1684 (the twenty-third year of Emperor Kangxi of Qing Dynasty), the Qing government opened the sea ban and established four customs offices: Yue (in Guangzhou), Min (in Xiamen), Jiang (in Yuntai Mountain, now near Lianyungang) and Zhe (in Ningbo). In 1685 (24th year of Emperor Kangxi), the Qing government established the first batch of customs in China (Yue, Min, Jiang, Zhe), of which Yue Customs was located at Tianzi Wharf next to Haizhu Square in Guangzhou and Yue Custom has been established. It is nominally specialized in foreign trade and tariff levy. In fact, tax revenue is managed by Thirteen Factories and undertakes contracted projects. These include the business of customs declaration and tax payment, and the purchase and sale of goods.

${ }^{10}$ On September 3, 1861(July 29, Xianfeng 11), Britain and France
}

Figure 5(Right) Yi Guan Area drawing by Prof. Zhaoxuan Zeng $^{8}$

The development of Yi Guan Area has experienced a development period of about 170 years from $1685^{9}$ to $1856^{10}$. According to the research of Prof. Zhaoxuan Zeng, the specific location of the Thirteen Factories Yi Guan Area is in the south of the Thirteen Streets, in the north of the Pearl River bank, the Xihao Road in the east and Lianxing Street in the west. It is now the area of Guangzhou Cultural Park, Thirteen Streets, Renmin South Road and Renji Road (Figure 4). According to the position of the river shoreline in each period in ${ }^{11}$ Prof. Zeng's "Thirteen Factories Distribution Map" (Figure 5), we could see that Yi Guan Area of the Thirteen Factories continues to expand southward along with the southern migration of the Pearl River shoreline. The riverbank lines of 1777 , 1903 and 1907 are well documented and marked in a clear position. According to the characteristics of architectural style evolution, Prof. Changxin Peng defines the first period of architectural form evolution of Yi Guan Area as "1748-1822"12. At the same time, there are a lot of image data about the business district around 1800 (Table 1). Therefore, the historical time for digitizing the business district will be selected between 1777 and 1822 .

Table 1. Image of the British Factory during 1780 and 1810

\begin{tabular}{|c|c|c|c|}
\hline No. & Time & Image & Sight analysis \\
\hline Figure $6^{13}$ & 1805 & & $\begin{array}{l}\text { From the } \\
\text { Southeast } \\
\text { look at the front } \\
\text { horizontal }\end{array}$ \\
\hline Figure $7^{14}$ & 1807 & & West facade \\
\hline Figure $8^{15}$ & 1810 & 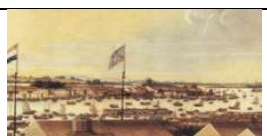 & $\begin{array}{l}\text { From the } \\
\text { Northeast } \\
\text { overlooking }\end{array}$ \\
\hline
\end{tabular}

The data of environmental land-forms first locate the corresponding land area on the Openstreetmap.org website. and relevant parameters are input to obtain the OSM data

forced the Qing government to sign the "Sand Rent Treaty."

11 Zeng Zhaoxuan, Zeng Xin, Zeng Xianshan. The Historical Geography of the Thirteen Factories Business District in Guangzhou -- The Sprout of China's Concession[J]. Guangzhou: Lingnan Culture and History, 01, 1999.

12 Changxin Peng. Modernity · Locality-Modern Transformation of Lingnan City and Architecture[M]. Shanghai: Tongji University Press, 2012. P46.

${ }^{13}$ View of the Canton Factories 1805-1806.View of the Pearl River Delta: Macau, Guangzhou and Hong Kong[M]. Printing Department, 2002.P161

${ }^{14}$ A Close of the Foreign Factories, 1807. ibid., P169.

15 Panoramic View of Canton Across the Rooftops of the Foreign Factories 1810, ibid., P171. 
file. ArcGIS can be used to interpret the current status of road network, traffic, lakes and other parameters, and can be converted into vector files on the modeling software. By combining the riverbank line drawn by Prof. Zeng of 1777 and the old map information of Qing Dynasty in Guangzhou with the current geographical range map, the environmental landscape map of the Thirteen Factories District that is closer to 1777 can be obtained step by step.

\subsection{D Modeling process}

The image modeling and virtual interaction of Yi Guan Area are carried out in a Cartesian coordinate system, so any object needs to have at least any factor of 3D. In fact, any 3D object will have one or thousands of polygons. NURBS software can be used for curved surfaces. It can distort, stretch or edit objects like clay in an organic way. It is suitable for constructing curved surfaces with complex curves.

Base on the previous discussion, the square terrace of the British Factory in 1805 is selected from the historical materials of Yi Guan Area for the practice of modeling cases. The first is to collect images of the front porch of the British Factory in different years (Table 1), and to analyze the vanishing point of sight with perspective method and proportional method; the second is to calculate the plane size of the square terrace according to the scale of the British Factory in the map of 1840 is near $22.2 \mathrm{~m} \mathrm{X} 22.2$ $\mathrm{m}$; The third is to calculate the height of the architecture according to the vanishing point of the British Factory perspective (Figure 6) in 1805.

In Table 1, the architectural form of the British Factory in the Thirteen Factories along the river in 1780, 1785 and 1810 is compared.

The structure of the Opening on three sides, four depth and double slope top has been maintained. Combining the OSM documents of the current topographic conditions with the shoreline of the Pearl River, it can be deduced that the architectural boundary and facade of the British Pavilion hall between 1760 and 1805 have not changed much.
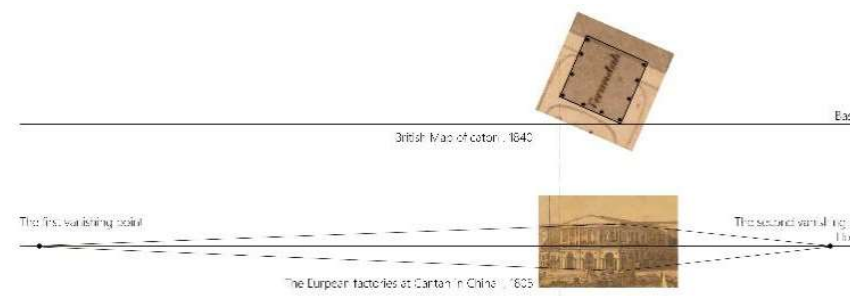

Figure 6. Perspective Analysis of the Front Gallery of the 1805 British Factory

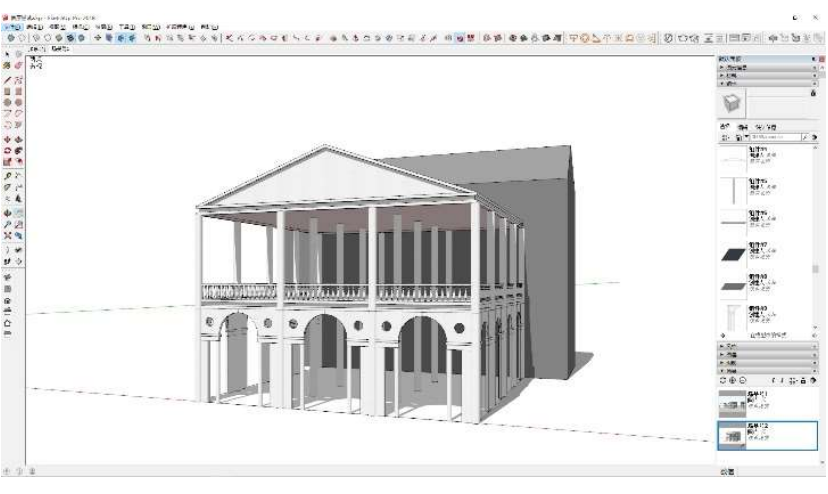

Figure 7. The geometric model of the front porch space of British factory in 1850

The spatial model of the front porch of the British Pavilion is derived from the collection, calculation and transformation of the image data of the top view, horizontal view, flat view and western elevation of the British Factory in the images of different periods in Table 1. The architecture front porch is a square building with two floors, north and south with three openings (Figure 7), east and west with four openings. The first floor of the building's façade uses a typical Palladian motif, each of which follows a 1:1 aesthetic ratio. This building facade style reflects the aesthetic appeal of British architecture from 1750 to 1810 .

\subsection{D texture optimization}

The modeling process of ancient Chinese architecture is usually divided into five layers: beam frame layer, tile surface layer, column foundation layer, bucket arch layer and decoration layer. In Qing Dynasty, the architectural style of Yi Guan Area in Canton tends to be European style. Important components such as columns, arch and pediment are very different from those of Chinese ancient architecture. According to the function of architecture components and modeling process, we set up the database of Yi Guan Area into three categories: structure component factor, maintenance component factor and decoration component factor (Figure 8). In this way, a series of next level databases will be established, and the corresponding material database will be established according to this classification.

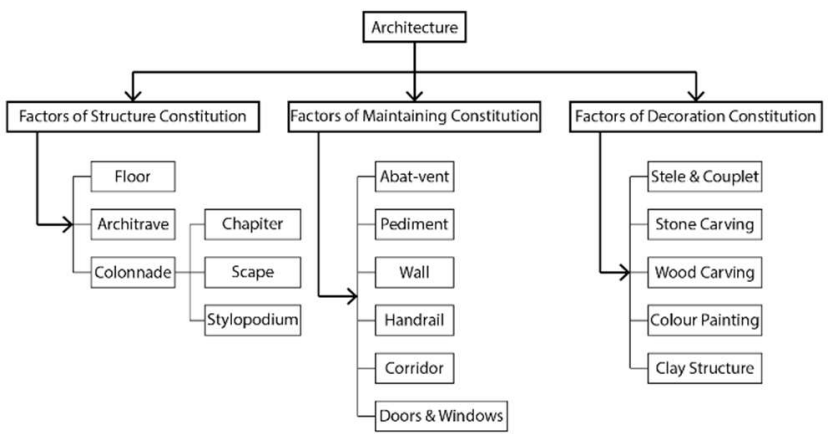

Figure 8. Architectures of The Thirteen Factories modelling databases 
The perfect visual art effect is one of the goals pursued by virtual interactive technology. Texture mapping can make the model show more details. Commonly used is two-dimensional graphics using U-V coordinates corresponding to two-dimensional texture $\mathrm{X}$ and $\mathrm{Y}$ coordinates for model matching. Sometimes we can also use Right Hemisphere's Deep U V to expand the U-V coordinates, placing them flat on texture maps for easy texture production. ${ }^{16}$

Texture optimization can enhance the aesthetic sense of the model and make the historical architecture look more real and beautiful. Texture optimization includes baking techniques and transparent map processing techniques. Baking map technology is to paste the map of light information into the scene. The advantage of this method is that it can reduce the system memory used to calculate the lighting when browsing the system, which is beneficial for the system resources to be used to draw triangles. Meanwhile, the rendered texture with lighting information can enhance the sense of reality of the scene. There are two types of baking maps: complete map and lighting-map.

Ancient Chinese architecture is especially concerned with decoration and ornament. Although the images and colors chosen vary according to the location and the nature of the components, all parts or components of the architecture should be beautified Such as ancient buildings' roof eaves, porch chairs, hollow carved windows and so on. This kind of decoration component is compact and diverse, and the workload of physical modeling is large, which will occupy memory space. If transparent mapping is used instead of physical model, it can not only express rich visual details, but also simplify the model data. For example, the door, window, wall and other components of the architecture's facade need only two triangular patches when constructing the 3D model, which can be used to give a hollow transparent map.

The export paintings the Thirteen Factories of Guangzhou are painted by Chinese painters and specially for export to Europe and the United States for oil painting, gouache and watercolor painting. ${ }^{17}$ Among them, Western painting techniques are adopted to varying degrees, and the content of the painting reflects the social, historical and living scenes of the time, which are the main source and research basis of the texture material library.

\section{Conclusion}

Based on the case of digitization of Yi Guan Area in Qing Dynasty, this paper discusses the digitization method and application of 3D virtual interaction of non-physical historical architecture from 3D information extraction and interpretation, 3D modeling and texture mapping. It not only guarantees the display speed, but also achieves better architectural art expression effect. It is hoped that the optimized modeling of the subject and the interior of the architecture can be extended to the streets, gardens and rivers of the whole Thirteen Factories District, and try to reproduce the splendid Thirteen Factories District of Canton in Qing Dynasty vividly and completely. Today, the cultural industry and the information industry are two "super industries forming an integral part" in modern society. Information technology fundamentally changes and guides the production, dissemination and consumption of cultural products. The use of information technology can improve the originality of architectural cultural heritage, enhance the competitiveness and vitality of architectural cultural heritage, better promote the transformation of cultural resources into economic advantages, create huge economic and social benefits, and promote social and economic development better and faster.

\section{References}

1. Tang Guoan(2006). ArcGIS Geographic Information System Spatial Analysis Experimental Course. Beijing: Science Press.

2. Hu Xiaoqiang(2005). Virtual Reality Technology. Beijing: Beijing University of Posts and Telecommunications Press.

3. Van Dyke Paul A. (2017), The Hume Scroll of 1772 and the Faces behind the Canton Factories. Review of Culture, International Edition 54,2017.

4. Van Dyke, Paul A.; Mok, Maria Kar-wing (2015), Images of the Canton Factories 1760-1822: Reading History in Art, Hong Kong: Hong Kong University Press.

5. Farris, Johnathan A. (2007). "Thirteen Factories of Canton". Buildings \& Landscapes, Vol. 14.

6. Perdue, Peter C. (2011). "Rise \& Fall of the Canton Trade System” . MIT Visualizing Cultures. 2014.

7. Hong Kong Museum of Art (2002). View of the Pearl River Delta: Macau, Guangzhou and Hong Kong. Printing Department.

8. Changxin Peng(2012). Modernity LocalityModern Transformation of Lingnan City and Architecture. Shanghai: Tongji University Press, 2012. P46. (in Chinese)

\footnotetext{
16 MattD.Dunbar.3D Visualization for the analysis of forest cover change [J].GeocartoInternational,2004.19(2):103-112

Lin You. Discussion on the Modern Significance of the Export
}

Paintings of the Thirteen Factories Business District in Guangzhou in the 18th and 19th Centuries[J]. Brand: Market Research, 2015 (01). 